\title{
ON ROTH'S PSEUDO EQUIVALENCE OVER RINGS*
}

\author{
R.E. HARTWIG ${ }^{\dagger}$ AND PEDRO PATRICIO $\ddagger$
}

\begin{abstract}
.
We characterize the pseudo-equivalence of a block lower triangular matrix $T=\left[T_{i j}\right]$ over a regular ring, and its block diagonal matrix $D(T)=\left[T_{i i}\right]$, in terms of suitable Roth consistency conditions. The latter can in turn be expressed in terms of the solvability of certain matrix equations of the form $T_{i i} X-Y T_{j j}=U_{i j}$.
\end{abstract}

Key words. Regular rings, Roth equivalence.

AMS subject classifications. 16A30, 15A21

1. Introduction and Definitions. Let $\mathcal{R}$ be a ring with unity 1 , let $\mathcal{R}_{m \times n}$ be the set of $m \times n$ matrices over $\mathcal{R}$, and shorten $\mathcal{R}_{n \times n}$ to $\mathcal{R}_{n}$. Throughout all our rings will have an identity.

An element $a \in \mathcal{R}$ is said to be regular if $a=a x a$, for some $x$, which is denoted by $x=a^{-}$. $\mathcal{R}$ is said to be regular if all of its elements are regular. A reflexive inverse $a$ is an element $x$, such that $a x a=a, x a x=x$. We shall denote such an inverse of $a$ by $a^{+}$. The sets of inner and reflexive inverses of $a$, if any, will be respectively denoted by is $a\{1\}$ and $a\{1,2\}$.

Definition 1.1. $m, n \in \mathcal{R}$ are pseudo-equivalent, $m \approx n$, provided there exist regular elements $p, q$ and $p^{-}, q^{-}$such that

$$
n=p m q, m=p^{-} n q^{-} .
$$

We may without loss of generality replace the inner inverses $p^{-}, q^{-}$by reflexive inverses $p^{+}, q^{+}$.

A ring $\mathcal{R}$ is called (von Neumann) finite if $a b=1$ implies $b a=1$, and it is called stably finite if $\mathcal{R}_{n}$ is finite for all $n \in \mathbb{N}$.

A ring $\mathcal{R}$ is called unit regular if for every $a$ in $R$, aua $=a$, for some unit $u$ in $R$.

When $p>q$ then $R_{p}$ finite implies that $R_{q}$ is finite.

Matrices $A$ and $B$ are said to be equivalent, denoted by $A \sim B$, if $A=P B Q$ for some invertible matrices $P, Q$. Likewise, matrices $A$ and $B$ are said to be pseudoequivalent, denoted by $A \approx B$, if $A=P B Q$ and $B=P^{+} B Q^{+}$for some square matrices $P$ and $Q$, with reflexive inverses $P^{+}, Q^{+}$.

If $A=\left[A_{i j}\right]$ is a block matrix over $\mathcal{R}$ with $A_{i j}$ of size $p_{i} \times q_{j}$, and $i, j=1, \ldots, n$, then $A_{k}$ with $k \leq n$, is the leading principal block-submatrix $\left[A_{i j}\right]$ with $i, j=1, \ldots, k$. The trailing principal submatrix is given by $\hat{A}_{k}=\left[A_{i j}\right]$ with $i, j=n-k+1, \ldots, n-1, n$,

\footnotetext{
* Received by the editors on ... Accepted for publication on .... Handling Editor: ...

$\dagger$ Mathematics Department, N.C.S.U. Raleigh, NC 27695-8205, U.S.A. (hartwig@unity.ncsu.edu).

‡Departamento de Matemática, Universidade do Minho, 4710-057 Braga, Portugal (pedro@math.uminho.pt). Member of the research unit CMAT - Centro de Matemática da Universidade do Minho, Portugal.
} 
i.e.,

$$
A=\left[\begin{array}{cc}
A_{k} & ? \\
? & \hat{A}_{n-k}
\end{array}\right]
$$

Definition 1.2. For a block matrix $A=\left[A_{i j}\right]$ we define its diagonal as the block matrix $D(A)=\operatorname{diag}\left(A_{11}, \ldots, A_{n n}\right)$. We further set $D_{k}(A)=\operatorname{diag}\left(A_{11}, \ldots, A_{k k}\right)$.

2. Roth Conditions. Consider the matrix

$$
M=\left[\begin{array}{cc}
A & 0 \\
B & D
\end{array}\right] .
$$

When the matrix equation $D X-Y A=B$ has a solution pair $(X, Y)$, onde checks that

$$
\left[\begin{array}{ll}
I & 0 \\
Y & I
\end{array}\right]\left[\begin{array}{ll}
A & 0 \\
B & D
\end{array}\right]\left[\begin{array}{ll}
I & 0 \\
-X & I
\end{array}\right]=\left[\begin{array}{ll}
A & 0 \\
0 & D
\end{array}\right]
$$

i.e., $M$ is equivalent to its block diagonal matrix $N=\operatorname{diag}(A, D)$.

On the other hand, for regular matrices $A$ and $D$, the consistency of $D X-Y A=B$ is equivalent to the condition $\left(1-D D^{-}\right) B\left(1-A^{-} A\right)=0$, for some and hence all $D^{-}, A^{-}$.

Given consistency, it was shown in [4] that over a regular ring, the general solution to $D X-Y A=B$ is given by

$$
\begin{aligned}
& X=D^{-} B+D^{-} Z A+\left(I-D D^{-}\right) W \\
& Y=-\left(I-D D^{-}\right) B A^{-}+Z-\left(I-D D^{-}\right) Z A A^{-},
\end{aligned}
$$

where $\mathrm{W}$ and $\mathrm{Z}$ are arbitrary.

In 1952, W.E. Roth proved the converse of 2.1 for matrices over a field $\mathbb{F}$ [16], i.e.,

(2.2) $D X-Y A=B$ has a solution pair if and only if $\left[\begin{array}{cc}A & 0 \\ B & D\end{array}\right] \sim\left[\begin{array}{cc}A & 0 \\ 0 & D\end{array}\right]$.

A ring $R$ is said to have Roth's equivalence property if the equivalence 2.2 is valid for all matrices over $R$. Roth's equivalence property was extended in [10], where it was shown that over a unit regular ring,

$$
\left[\begin{array}{ll}
a & 0 \\
b & d
\end{array}\right] \sim\left[\begin{array}{ll}
a & 0 \\
0 & d
\end{array}\right] \Leftrightarrow d x-y a=b \text { has a solution pair }(x, y) \text {. }
$$

This result implies that such rings must be finite, and have Roth's equivalence property.

This result was later extended to regular rings by Guralnick [6], who showed that over a regular ring, Roth's equivalence property holds if and only if $\mathcal{R}$ is stably finite. In a 
parallel paper [7], Gustafson proved that a commutative ring also must have Roth's equivalence property.

We shall show that in Roth's equivalence property, we may replace equivalence by pseudo-equivalence, provided the diagonal blocks $A_{i}$ are regular and $\mathcal{R}_{\tau}$ is finite, for suitable $\tau$.

3. Lemmata. We begin by deriving some simple consequences of pseudo equivalence.

LEMMA 3.1. If $n \approx m$, then

(i) $\approx$ is symmetric.

(ii) $m$ is regular if and only if $n$ is regular.

(iii) $n R=p m R, R n=R m q$.

(iv) $m R \cong n R$.

Proof. (iv) Let $\phi(m x)=n q x$. Then $n q x_{1}=n q x_{2} \Rightarrow m x_{1}=p n q x_{1}=p n q x_{2}=$ $m x_{2}$. Also for any $s, n s=n q q^{+} s=\phi\left(m q^{+} s\right)$. As such $\phi$ is a one-one onto module isomorphism, and therefore $m R$ and $n R$ are isomorphic.

We may at once apply these to the matrix rings over $R$, and state

Corollary 3.2. If $M \approx D$ and $M \sim M^{\prime}$, then $R(D) \cong R\left(M^{\prime}\right)$.

The following lemma was proved in [11] and characterizes the finiteness of $\mathcal{R}_{n}$.

Lemma 3.3. Let $e, f \in \mathcal{R}$ with $e^{2}=e$ and $f^{2}=f$. The following conditions are equivalent:

1. $\mathcal{R}$ is finite.

2. $e \mathcal{R} \subseteq f \mathcal{R}, e \mathcal{R} \cong f \mathcal{R} \Longrightarrow e \mathcal{R}=f \mathcal{R}$.

3. $\mathcal{R} e \subseteq \mathcal{R} f, \mathcal{R} e \cong \mathcal{R} f \Longrightarrow \mathcal{R} e=\mathcal{R} f$.

We shall apply this to regular matrix rings.

A key result in our reduction is the following "corner" lemma.

LEMMA 3.4.

(a) When $y d^{+}=0$ then $\left[\begin{array}{cc}a & 0 \\ b & d+y\end{array}\right] \sim\left[\begin{array}{cc}a & 0 \\ \left(1-d d^{+}\right) b\left(1-a^{+} a\right) & d\end{array}\right]$.

(b) If $M=\left[\begin{array}{ll}a & 0 \\ r & d\end{array}\right]$, with $r=\left(1-d d^{+}\right) b\left(1-a^{+} a\right)$, then there exists a $1-2$ inverse

$$
M^{+}=\left[\begin{array}{cc}
a^{+} & \left(1-a^{+} a\right) r^{+}\left(1-d d^{+}\right) \\
0 & d^{+}
\end{array}\right]
$$

such that

$$
\begin{aligned}
& M M^{+}=\left[\begin{array}{cc}
a a^{+} & 0 \\
0 & d d^{+}+r r^{+}\left(1-d d^{+}\right)
\end{array}\right], \\
& M^{+} M=\left[\begin{array}{cc}
a^{+} a+\left(1-a^{+} a\right) r^{+} r & 0 \\
0 & d^{+} d
\end{array}\right] .
\end{aligned}
$$


Proof. (a)

$$
\left[\begin{array}{cc}
1 & 0 \\
-b a^{+} & 1
\end{array}\right]\left[\begin{array}{cc}
a & 0 \\
b & d+y
\end{array}\right]\left[\begin{array}{cc}
1 & 0 \\
-d^{+} b\left(1-a^{+} a\right) & 1
\end{array}\right]=\left[\begin{array}{cc}
a & 0 \\
\left(1-d d^{+}\right) b\left(1-a^{+} a\right) & d
\end{array}\right] \text {. }
$$

(b) This fundamental result was shown in [9], pg 211 eq. (3.5).

4. The Cornered Canonical Forms. We next turn to the block triangular case. Let $R$ be a regular ring and let

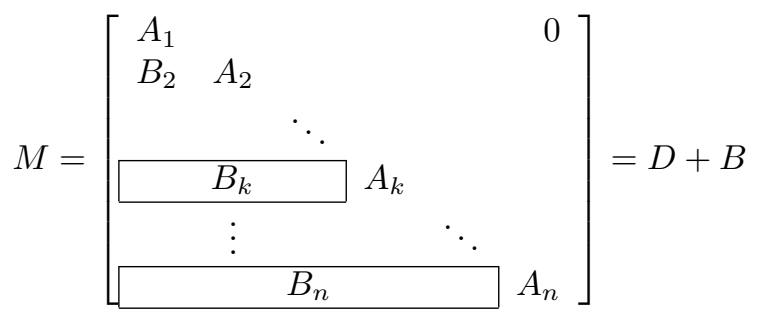

be a lower triangular block matrix with $D=D(M)=\operatorname{diag}\left(A_{1}, . ., A_{n}\right)$.

We shall assume that $A_{i}$ is $p_{i} \times q_{j}$ and $M$ is $p \times q$, where $p=\sum_{i=1}^{n} p_{i}$ and $q=\sum_{j=1}^{n} q_{j}$.

Our aim is to address the question of how to characterize $M \sim D$ and $M \approx D$. The former was done in [12], with aid of the canonical form

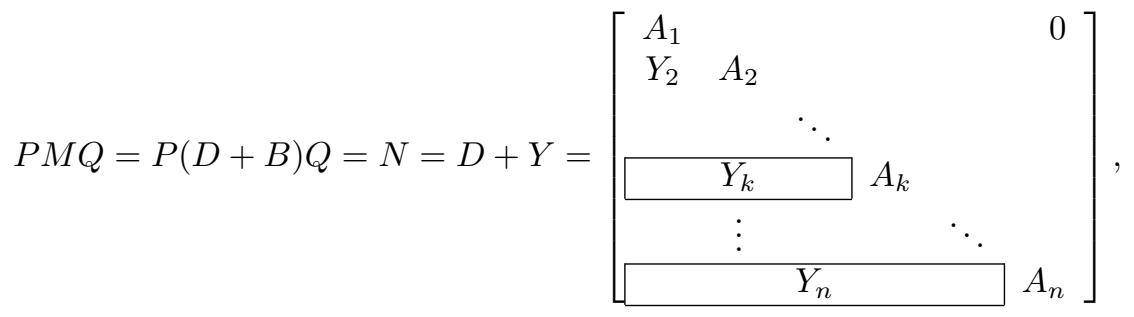

where

$$
\begin{aligned}
P & =P_{n}\left[\begin{array}{cc}
P_{n-1} & 0 \\
0 & 1
\end{array}\right] . .\left[\begin{array}{cc}
P_{2} & 0 \\
0 & I_{n-2}
\end{array}\right]=\left[\begin{array}{cc}
\Delta_{k} & 0 \\
0 & I_{n-k}
\end{array}\right], \\
Q & =\left[\begin{array}{cc}
Q_{2} & 0 \\
0 & I_{n-2}
\end{array}\right] . .\left[\begin{array}{cc}
Q_{n-1} & 0 \\
0 & 1
\end{array}\right] Q_{n}=\left[\begin{array}{cc}
\pi_{k} & 0 \\
0 & I_{n-k}
\end{array}\right], \\
\Delta_{k} & =P_{k}\left[\begin{array}{cc}
P_{k-1} & 0 \\
0 & 1
\end{array}\right] \ldots\left[\begin{array}{cc}
P_{2} & 0 \\
0 & I_{k-2}
\end{array}\right]=P_{k}\left[\begin{array}{cc}
\Delta_{k-1} & 0 \\
0 & 1
\end{array}\right]
\end{aligned}
$$

and

$$
\pi_{k}=\left[\begin{array}{cc}
Q_{2} & 0 \\
0 & I_{k-2}
\end{array}\right] \ldots\left[\begin{array}{cc}
Q_{k-1} & 0 \\
0 & 1
\end{array}\right] Q_{k}=\left[\begin{array}{cc}
\pi_{k-1} & 0 \\
0 & 1
\end{array}\right]
$$


In these expressions

$$
\begin{aligned}
& P_{k}=\left[\begin{array}{cc}
I_{k-1} & 0 \\
-B_{k} \pi_{k-1} D_{k-1}^{+} & 1
\end{array}\right] \text { and } \\
& Q_{k}=\left[\begin{array}{cc}
I_{k-1} & 0 \\
-A_{k}^{+} B_{k} \pi_{k-1}\left(I-D_{k-1}^{+} D_{k-1}\right) & 1
\end{array}\right]=\left[\begin{array}{cc}
I_{k-1} & 0 \\
-\mathbf{q}_{k} & 1
\end{array}\right]
\end{aligned}
$$

are both $k \times k$.

In addition, the submatrices $Y_{k}$ of $N$, are defined by

$$
Y_{k}=\left(1-A_{k} A_{k}^{+}\right) B_{k} \pi_{k-1}\left(I-D_{k-1}^{+} D_{k-1}\right) .
$$

The reduction as given in 4.2 is equivalent to the "horizontal" reduction

$$
V^{-1} M\left(U^{-1} Z\right)=N=D+Y
$$

in which $U=I+D^{+} B, V=I+B D^{+}$and $Z=I+D^{+} B D D^{+}$are all invertible.

In order to solve the pseudo-equivalence problem, we shall need a second parallel canonical form, which again uses Lemma 3.4,

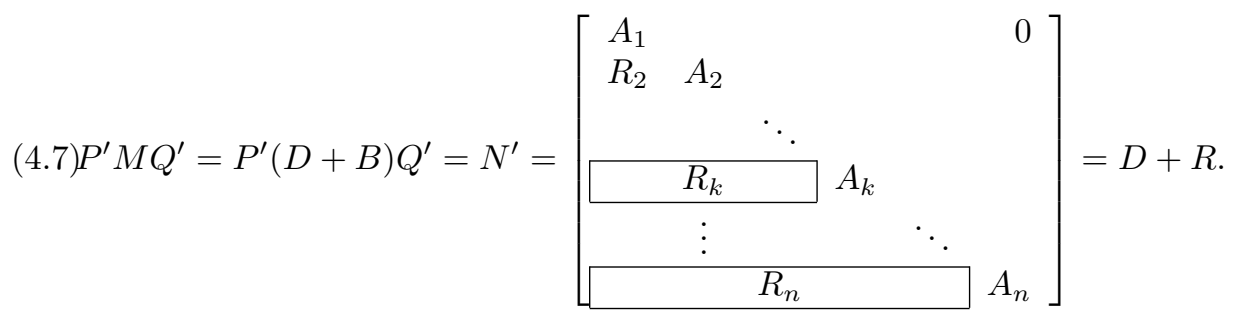

The steps in this reduction are identical to those used to obtain $N$, except that we replace $D_{k}$ by the principal block $N_{k}^{\prime}$ at each stage. This gives

$$
\begin{aligned}
& P^{\prime}=P_{n}^{\prime} \ldots\left[\begin{array}{cc}
P_{k}^{\prime} & 0 \\
0 & I_{n-k}
\end{array}\right] \ldots\left[\begin{array}{cc}
P_{2}^{\prime} & 0 \\
0 & I_{n-2}
\end{array}\right]=\left[\begin{array}{cc}
\Delta_{k}^{\prime} & 0 \\
0 & I_{n-k}
\end{array}\right], \\
& Q^{\prime}=\left[\begin{array}{cc}
Q_{2}^{\prime} & 0 \\
0 & I_{n-2}
\end{array}\right] \ldots\left[\begin{array}{cc}
Q_{k}^{\prime} & 0 \\
0 & I_{n-k}
\end{array}\right] \ldots Q_{n}^{\prime}=\left[\begin{array}{cc}
\pi_{k}^{\prime} & 0 \\
0 & I_{n-k}
\end{array}\right],
\end{aligned}
$$

and

$$
\begin{gathered}
\Delta_{k}^{\prime}=P_{k}^{\prime}\left[\begin{array}{cc}
P_{k-1}^{\prime} & 0 \\
0 & I
\end{array}\right] \cdots\left[\begin{array}{cc}
P_{2}^{\prime} & 0 \\
0 & I_{k-2}
\end{array}\right] \text { and } \\
\pi_{k}^{\prime}=\left[\begin{array}{cc}
Q_{2}^{\prime} & 0 \\
0 & I_{k-2}
\end{array}\right] \cdots\left[\begin{array}{cc}
Q_{k-1}^{\prime} & 0 \\
0 & I
\end{array}\right] Q_{k}^{\prime} .
\end{gathered}
$$


In these products,

$$
\begin{gathered}
P_{k}^{\prime}=\left[\begin{array}{cc}
I_{k-1} & 0 \\
-B_{k} \pi_{k-1}^{\prime}\left(N_{k-1}^{\prime}\right)^{+} & I
\end{array}\right] \text { and } \\
Q_{k}^{\prime}=\left[\begin{array}{cc}
I_{k-1} & 0 \\
-A_{k}^{+} B_{k} \pi_{k-1}^{\prime}\left(I-\left(N_{k-1}^{\prime}\right)^{+} N_{k-1}^{\prime}\right) & I
\end{array}\right]=\left[\begin{array}{cc}
I_{k-1} & 0 \\
-\mathbf{q}_{k}^{\prime} & I
\end{array}\right]
\end{gathered}
$$

are both are $k \times k$.

It should be noted that

$$
\pi_{2}^{\prime}=\pi_{2}=Q_{2}=\left[\begin{array}{cc}
I & 0 \\
-B_{2} A_{1}^{+} & I
\end{array}\right]
$$

and

$$
\Delta_{2}^{\prime}=\Delta_{2}=\left[\begin{array}{cc}
I & 0 \\
-A_{2}^{+} B_{2}\left(I-A_{1}^{+} A_{1}\right) & I
\end{array}\right] .
$$

The submatrices $R_{k}$ of $N^{\prime}$ in 4.7 are defined by

$$
R_{k}=\left(1-A_{k} A_{k}^{+}\right) B_{k} \pi_{k-1}^{\prime}\left(I-\left(N_{k-1}^{\prime}\right)^{+} N_{k-1}^{\prime}\right) .
$$

There does not seem to be an obvious "horizontal" reduction (using $D, B$ and (.) $)^{+}$ that is equivalent to this "total" block reduction!

We next take advantage of the special form of $N^{\prime}$. Using Lemma 3.4 we obtain the following

TheOREM 4.1. Let $N^{\prime}$ be as in 4.7. Then there exists a reflexive inverse $\left(N^{\prime}\right)^{+}$ such that

$N^{\prime}\left(N^{\prime}\right)^{+}=\operatorname{diag}\left(A_{1} A_{1}^{+}, A_{2} A_{2}^{+}+R_{2} R_{2}^{+}\left(1-A_{2} A_{2}^{+}\right), \ldots, A_{n} A_{n}^{+}+R_{n} R_{n}^{+}\left(1-A_{n} A_{n}^{+}\right)\right)=\mathfrak{D}$

Proof. If $N_{k}^{\prime}=\left[\begin{array}{cc}N_{k-1}^{\prime} & 0 \\ R_{k} & A_{k}\end{array}\right]$ then by Lemma 3.4 we can find a reflexive inverse

$$
\left(N^{\prime}\right)_{k}^{+}=\left[\begin{array}{cc}
\left(N^{\prime}\right)_{k-1}^{+} & \left(I-\left(N^{\prime}\right)_{k-1}^{+} N_{k-1}^{\prime}\right) R_{k}^{+}\left(1-A_{k} A_{k}^{+}\right) \\
0 & A_{k}^{+}
\end{array}\right] .
$$

Recalling that $A_{k}^{+} R_{k}=0$ and $R_{k}\left(N^{\prime}\right)_{k-1}^{+}=0$, we may conclude that

$$
\left(N^{\prime}\right)_{k}\left(N^{\prime}\right)_{k}^{+}=\left[\begin{array}{cc}
\left(N^{\prime}\right)_{k-1}\left(N^{\prime}\right)_{k-1}^{+} & 0 \\
0 & A_{k} A_{k}^{+}+R_{k}\left(R_{k}\right)^{+}\left(1-A_{k} A_{k}^{+}\right)
\end{array}\right]
$$

We note in passing that $R_{k} D_{k}^{+} \neq 0$ in general.

It now follows by induction, that if $N_{k-1}^{\prime}\left(N^{\prime}\right)_{k-1}^{+}$is diagonal, then so is $N_{k}^{\prime}\left(N^{\prime}\right)_{k}^{+}$, and has the form

$N_{k}^{\prime}\left(N^{\prime}\right)_{k}^{+}=\operatorname{diag}\left[A_{1} A_{1}^{+}, A_{2} A_{2}^{+}+R_{2}\left(R_{2}\right)^{+}\left(1-A_{2} A_{2}^{+}\right), \ldots, A_{k} A_{k}^{+}+R_{k}\left(R_{k}\right)^{+}\left(1-A_{k} A_{k}^{+}\right)\right]$ for $k=1, \ldots, n$. 
When $k=n$, we arrive at $\mathfrak{D}=N_{n}^{\prime}\left(N^{\prime}\right)_{n}^{+}$, as desired.

Because the product of $A_{k} A_{k}^{+}$and $I-A_{k} A_{k}^{+}$is zero, we see that

$$
R\left[A_{k} A_{k}^{+}+R_{k}\left(R_{k}\right)^{+}\left(1-A_{k} A_{k}^{+}\right)\right]=R\left[A_{k} A_{k}^{+}\right] \dot{+} R\left[R_{k}\left(R_{k}\right)^{+}\left(1-A_{k} A_{k}^{+}\right)\right]
$$

as an internal direct sum, and hence that

$$
R\left(A_{k}\right)=R\left(A_{k} A_{k}^{+}\right) \subseteq R\left[A_{k} A_{k}^{+}+R_{k}\left(R_{k}\right)^{+}\left(1-A_{k} A_{k}^{+}\right)\right] .
$$

This allows us to conclude that

Corollary 4.2 .

$$
R(D)=\left[\begin{array}{c}
R\left(A_{1}\right) \\
\vdots \\
R\left(A_{n}\right)
\end{array}\right] \subseteq\left[\begin{array}{c}
R\left(A_{1}\right) \\
R\left(A_{2}\right)+R\left[R_{2}\left(R_{2}\right)^{+}\left(1-A_{2} A_{2}^{+}\right)\right] \\
\vdots \\
R\left(A_{n}\right) \dot{+} R\left[R_{n}\left(R_{n}\right)^{+}\left(1-A_{n} A_{n}^{+}\right)\right]
\end{array}\right]=R(\mathfrak{D})=R\left(N_{n}^{\prime}\right)
$$

We may now combine Corollaries 3.2 and 4.2 to conclude that

(i) $R(D) \subseteq R(\mathfrak{D})$.

(ii) $R(M) \cong R\left(N^{\prime}\right)=R\left[N^{\prime}\left(N^{\prime}\right)^{+}\right]=R(\mathfrak{D})$.

(iii) If $M \approx D$ then $R(D) \cong R(M) \cong R(\mathfrak{D})$.

Now if $R_{p \times p}$ is finite then by Theorem 1 of [10], we may conclude that $R(D)=R(\mathfrak{D})$ and thus $R\left[R_{k} R_{k}^{+}\left(1-A_{k} A_{k}^{+}\right)\right]=0$. This means that $R_{k}=R_{k}\left(R_{k}\right)^{+} R_{k}=0$, for $k=1,2, \ldots, n$ and we have the Roth Consistency Conditions $R=0$, i.e.,

$$
R_{k}=\left(1-A_{k} A_{k}^{+}\right) B_{k} \pi_{k-1}^{\prime}\left(I-\left(N^{\prime}\right)_{k-1}^{+} N_{k-1}^{\prime}\right)=0 .
$$

In order to relate these conditions to the condition that $Y=0$, we shall need the following

Lemma 4.3. Let $N^{\prime}$ be as in 4.7. For each $1 \leq t \leq n$, the following are equivalent.

(i) $N_{k}^{\prime}\left(N^{\prime}\right)_{k}^{+}=D_{k} D_{k}^{+}, k=1, \ldots, t$.

(ii) $R_{k}=0, k=2, \ldots, t$.

(iii) $\left(N_{k}^{\prime}\right)^{+} N_{k}^{\prime}=D_{k}^{+} D_{k}, k=1, \ldots, t$.

(iv) $Y_{k}=0, k=2, \ldots, t$.

Proof. We shall use induction in all four cases.

(i) $\Rightarrow$ (ii) From Theorem 4.1 we see that for $k \leq t$,

$$
N_{k}^{\prime}\left(N^{\prime}\right)_{k}^{+}=\left[\begin{array}{cc}
\left(N^{\prime}\right)_{k-1}\left(N^{\prime}\right)_{k-1}^{+} & 0 \\
0 & A_{k} A_{k}^{+}+R_{k}\left(R_{k}\right)^{+}\left(1-A_{k} A_{k}^{+}\right)
\end{array}\right] .
$$

If this equals $D_{k} D_{k}^{+}$, then we must have $R_{k}\left(R_{k}\right)^{+}\left(1-A_{k} A_{k}^{+}\right)=0$ for $k \leq t$. Postmultiplication by $B_{k} \pi_{k-1}^{\prime}\left(I-\left(N^{\prime}\right)_{k-1}^{+} N_{k-1}^{\prime}\right)$ then shows that $R_{k}=0$.

(ii) $\Rightarrow$ (i). Indeed, if $N_{k-1}^{\prime}\left(N^{\prime}\right)_{k-1}^{+}=D_{k-1} D_{k-1}^{+}$then setting $R_{k}=0$ in 4.12 , we see that

$$
N_{k}^{\prime}\left(N^{\prime}\right)_{k}^{+}=\left[\begin{array}{cc}
N_{k-1}^{\prime}\left(N^{\prime}\right)_{k-1}^{+} & 0 \\
0 & A_{k} A_{k}^{+}
\end{array}\right]=\left[\begin{array}{cc}
D_{k-1} D_{k-1}^{+} & 0 \\
0 & A_{k} A_{k}^{+}
\end{array}\right]=D_{k} D_{k}^{+} .
$$


(ii) $\Rightarrow$ (iii). One checks that $N_{2}^{+} N_{2}=D_{2}^{+} D_{2}$. Next we assume it holds for $k=$ $r-1$. Then from Lemma 3.4 we see that for $N_{r}^{+} N_{r}=\left[\begin{array}{cc}N_{r-1}^{+} N_{r-1} & 0 \\ 0 & A_{r}^{+} A_{r}\end{array}\right]=$ $\left[\begin{array}{cc}D_{r-1}^{+} D_{r-1} & 0 \\ 0 & A_{r}^{+} A_{r}\end{array}\right]=D_{r}^{+} D_{r}$.

(iii) $\Rightarrow$ (ii). If $N_{k}^{+} N_{k}=\left[\begin{array}{cc}N_{k-1}^{+} N_{k-1}+\left(I-N_{k-1}^{+} N_{k-1}\right) R_{k}^{+} R_{k} & 0 \\ 0 & A_{k}^{+} A_{k}\end{array}\right]=D_{k}^{+} D_{k}$, then

$$
N_{k-1}^{+} N_{k-1}+\left(I-N_{k-1}^{+} N_{k-1}\right) R_{k}^{+} R_{k}=D_{k-1}^{+} D_{k-1} .
$$

For $k=2$ we have $D_{2}^{+} D_{2}=N_{2}^{+} N_{2}$ and thus $\left(I-A_{1}^{+} A_{1}\right) R_{2}^{+} R_{2}=0$. This gives $R_{2}=0$. Assuming $R_{k}=0$ for $k<r$, shows that

$$
\begin{aligned}
D_{r}^{+} D_{r}=N_{r}^{+} N_{r} & =\left[\begin{array}{cc}
\left(N^{\prime}\right)_{r-1}^{+}\left(N^{\prime}\right)_{r-1}\left(I-N_{r-1}^{+} N_{r-1}\right) R_{r}^{+} R_{r} & 0 \\
0 & A_{r}^{+} A_{r}
\end{array}\right] \\
& =\left[\begin{array}{cc}
D_{r-1}^{+} D_{r-1}+\left(I-D_{r-1}^{+} D_{r-1}\right) R_{r}^{+} R_{r} & 0 \\
0 & A_{r}^{+} A_{r}
\end{array}\right] .
\end{aligned}
$$

This gives $\left(I-D_{r-1}^{+} D_{r-1}\right) R_{r}^{+} R_{r}=0$ and hence that $R_{r}=0$.

(ii) $\Rightarrow$ (iv). If $N_{k}^{+} N_{k}=D_{k}^{+} D_{k}$ for $k \leq t$ then $\pi_{k}^{\prime}=\pi_{k}$ and $R_{k}=Y_{k}$.

(iv) $\Rightarrow$ (ii) If $Y_{2}=0$ then clearly $R_{2}=0$. So assume that $R_{i}=Y_{i}=0$ for $i=$ $2, \ldots, k-1$. Then for these values of $i, N_{i}^{+} N_{i}=D_{i}^{+} D_{i}$ and $\pi_{i}^{\prime}=\pi_{i}$. Consequently $R_{k}=\left(I-A_{k} A_{k}^{+}\right) B_{k} \pi_{k-1}^{\prime}\left(I-N_{k}^{+} N_{k}\right)=\left(I-A_{k} A_{k}^{+}\right) B_{k} \pi_{k-1}\left(I-D_{k}^{+} D_{k}\right)=Y_{k}=0$.

We may now combine all the above in

TheOREM 4.4. Let $M$ be a block triangular matrix as in 4.1 and suppose that $R_{p \times p}$ is finite regular, then the following are equivalent:

(i) $M \sim D(M)$.

(ii) $M \approx D(M)$.

(iii) $R_{k}=0, k=2, \ldots, n$.

(iv) $Y_{k}=0, k=2, \ldots, n$.

5. Back to the Roth Conditions. Let us now turn the Roth conditions $Y=0$, into matrix equations. It should be noted that the equivalent condition $R=0$ is not so transparent. When $k=2$, we see that the first Roth consistency condition becomes

$$
R_{2}=\left(1-A_{2} A_{2}^{+}\right) B_{1}\left(I-A_{1}^{+} A_{1}\right)=0,
$$

which return us to $A_{2} X-Y A_{1}=B_{2}$. 
For general $k$, we recall the consistency condition [12]

$$
(I-E) B U^{-1}(I-F)=0
$$

in which $E=D D^{+}$and $F=D^{+} D$. Using path products this gives for the $(p, q)$ block,

$$
\left(I-A_{p} A_{p}^{+}\right)\left[\left(B U^{-1}\right)_{p q}\right]\left(I-A_{q}^{+} A_{q}\right)
$$

where

$$
\left(B U^{-1}\right)_{p q}=B_{p q}+\sum_{k=1}^{r}(-1)^{k} \sum_{p>i_{1}>i_{2}>. .>i_{k}>q} B_{p, i_{1}} A_{i_{1}}^{+} B_{i_{1}, i_{2}} A_{i_{2}}^{+} \ldots B_{i_{k-1}, i_{k}} A_{i_{k}}^{+} B_{i_{k} q} .
$$

This leads at once to the Roth-matrix consistency condition

$$
A_{p} X-Y A_{q}=\left(B U^{-1}\right)_{p q} \quad p, q=1, \ldots, n .
$$

We may now combine the above with the results of [6] and [7].

THEOREM 5.1. Let $R$ be a regular ring. The following are equivalent:

(i) $R$ has Roth's pseudo equivalence property, i.e., $M \approx D(M) \Rightarrow Y_{k}=0 \quad\left(R_{k}=0\right)$.

(ii) $R$ has Roth's equivalence property, i.e., $M \sim D(M) \Rightarrow Y_{k}=0 \quad\left(R_{k}=0\right)$.

(iii) $R$ is stably finite.

Proof. (i) $\Rightarrow$ (ii). This always holds.

(ii) $\Rightarrow$ (iii). This was shown in $[6]$.

(iii) $\Rightarrow$ (i). If $R$ is stably finite and regular, then $R_{N}$ is finite for all $N$. Now if in addition, $M \approx D(M)$ then by Theorem 4.4, we see that the Roth consistency conditions $Y_{k}=0$ hold for $k=1, \ldots, n$.

6. The Rowspace Case. The key condition in Theorem 4.1 was that $\mathcal{R}_{p}$ is finite. This condition can be weakened when $q<p$, to $\mathcal{R}_{q}$ being finite. To do this we have to repeat the above procedure with column spaces instead of row spaces.

The canonical form in this case can be obtained by using Lemma 3.4 starting from the lower right corner rather from the upper left corner and using trailing principal submatrices $\hat{M}_{k}$. Again we have two canonical forms. One involves $D_{k}$, which corresponds to the second horizontal factorization $W V^{-1} M U^{-1}=N=D+Y$. In this case it is more convenient to reverse the numbering of the blocks.

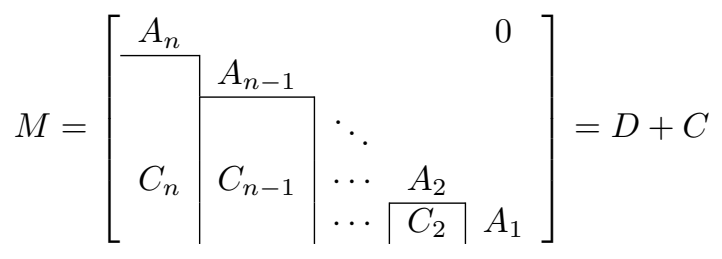

Even though the new Consistency conditions take a different form from the original Roth conditions, we shall show that we actually do get the same canonical matrix $M^{\prime}$ ! This will become clear once we identify this reduction with the factorization 
$W V^{-1} M U^{-1}=M^{\prime}$.

Our aim is to show that the reduction in this case can actually be obtained from the first procedure. We need two concepts.

Definition 6.1. If $A=\left[a_{i j}\right]$ is $m \times n$ then

(i) $\bar{A}=\left[\bar{a}_{i j}\right]$, where $a_{i j}=a_{m+1-j, n+1-i}$

(ii) $A^{\sim}=\left[a_{i j}^{\sim}\right]$ where in $a_{i j}^{\sim}$ we reverse all products, if any.

The former can be thought of as $\bar{A}=(F A F)^{T}$, where $F$ is the 'flip' matrix

$$
\left[\begin{array}{lll}
0 & & 1 \\
& . & \\
1 & & 0
\end{array}\right]
$$

provided we block transpose.

In particular if $D=\operatorname{diag}\left(A_{1}, . ., A_{n}\right)$ then $\bar{D}=\operatorname{diag}\left(A_{n}, . ., A_{1}\right)$.

We shall need

Theorem 6.2. Consider the matrices $A_{m \times k}, B_{k \times \ell}$ and $C_{\ell \times n}$ over an arbitrary ring. Then $\overline{(A B C)^{\sim}}=\bar{C} \bar{B} \bar{A}$

Proof. $(\bar{C} \bar{B} \bar{A})_{i j}=\sum_{u=1}^{\ell} \sum_{v=1}^{k}(\bar{C})_{i u}(\bar{B})_{u v}(\bar{A})_{v l}$ which in turn equals

$$
\sum_{u=1}^{\ell} \sum_{v=1}^{k} c_{\ell+1-u, n+1-i} b_{k+1-v, \ell+1-u} a_{m+1-j, k+1-v} .
$$

Now set $r=\ell+1-u$ and $s=k+1-v$. This gives

$$
(\bar{C} \bar{B} \bar{A})_{i j}=\sum_{r=1}^{\ell} \sum_{s=1}^{k} c_{r, n+1-i} b_{s, r} a_{m+1-j, s} .
$$

On the other hand, $(A B C)_{i j}=\sum_{s=1}^{k} \sum_{r=1}^{\ell} a_{i s} b_{s r} c_{r j}$ and hence

$$
\left(A B C^{\sim}\right)_{i j}=\sum_{s=1}^{k} \sum_{r=1}^{\ell} c_{r j} b_{s r} a_{i s} .
$$

Next we have $\left(\overline{(A B C)^{\sim}}\right)_{i j}=\sum_{s=1}^{k} \sum_{r=1}^{\ell} c_{r, n+1-i} b_{s r} a_{m+1-j, s}$, which is the $(i, j)$ entry in the RHS. $\mathrm{C}$

We shall apply this to the reduction $P^{\prime} M Q^{\prime}=N^{\prime}$, as given in 4.8-4.11.

Theorem 4.4 ensures that $\bar{Q}^{\prime} \bar{M} \bar{P}^{\prime}=\bar{N}^{\prime}$, in which

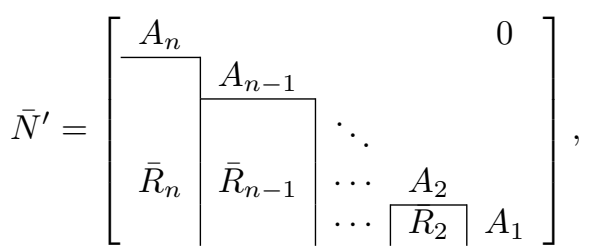


with $\bar{R}_{k}=\left(I-N_{k-1}^{\prime}\left(N^{\prime}\right)_{k-1}^{+}\right) \bar{\pi}_{k-1} C_{k}\left(I-A_{k}^{+} A_{k}\right)$ and

$$
\bar{P}^{\prime}=\left[\begin{array}{cc}
I_{n-2} & 0 \\
0 & \bar{P}_{2}^{\prime}
\end{array}\right] \ldots \bar{P}_{n}^{\prime}{ }_{n}=\left[\begin{array}{cc}
I_{n-k} & 0 \\
0 & \bar{\Delta}_{k}
\end{array}\right],
$$

with $\bar{\Delta}_{k}=\left[\begin{array}{cc}I_{k-2} & 0 \\ 0 & \bar{P}_{2}^{\prime}\end{array}\right] . .\left[\begin{array}{cc}1 & 0 \\ 0 & \bar{P}_{k-1}^{\prime}\end{array}\right] \bar{P}^{\prime}{ }_{k}$ and

$$
\bar{Q}^{\prime}=\bar{Q}_{n}^{\prime} \cdot .\left[\begin{array}{cc}
I_{n-2} & 0 \\
0 & \bar{Q}_{2}^{\prime}
\end{array}\right]=\left[\begin{array}{cc}
I_{n-k} & 0 \\
0 & \bar{\pi}_{k}
\end{array}\right]
$$

with $\bar{\pi}_{k}={\overline{Q^{\prime}}}_{k}^{\prime}\left[\begin{array}{cc}1 & 0 \\ 0 & {\overline{Q^{\prime}}}_{k-1}\end{array}\right] \ldots\left[\begin{array}{cc}I_{k-2} & 0 \\ 0 & {\overline{Q^{\prime}}}_{k-1}\end{array}\right]$, in which

$$
\bar{P}_{k}^{\prime}=\left[\begin{array}{cc}
1 & 0 \\
-\left(N^{\prime}\right)_{k-1}^{+} \bar{\pi}_{k-1}^{\prime} C_{k} & I_{k-1}
\end{array}\right]
$$

and

$$
{\overline{Q^{\prime}}}_{k}=\left[\begin{array}{cc}
1 & 0 \\
-\left(I-N_{k-1}^{\prime}\left(N^{\prime}\right)_{k-1}^{+}\right) \bar{\pi}_{k-1}^{\prime} C_{k} A_{k}^{+} & I_{k-1}
\end{array}\right] .
$$

To identify this canonical form we recall that

$$
\begin{gathered}
\Delta_{k+1}^{\prime}=P_{k+1}^{\prime}\left[\begin{array}{cc}
\Delta_{k}^{\prime} & 0 \\
0 & 1 I
\end{array}\right]=\left[\begin{array}{cc}
\Delta_{k}^{\prime} & 0 \\
-B_{k+1} \pi_{k} D_{k}^{+} \Delta_{k}^{\prime} & 1
\end{array}\right] \text { and } \\
\pi_{k+1}^{\prime}=\left[\begin{array}{cc}
\pi_{k}^{\prime} & 0 \\
0 & I
\end{array}\right] Q_{k+1}^{\prime}=\left[\begin{array}{cc}
\pi_{k}^{\prime} & 0 \\
-\mathbf{q}_{k+1}^{\prime} & 1
\end{array}\right]
\end{gathered}
$$

Again using Theorem 4.4 we now obtain

$$
{\overline{\pi^{\prime}}}_{k+1}=\left[\begin{array}{cc}
1 & 0 \\
-\overline{\mathbf{q}}^{\prime}{ }_{k+1} & \bar{\pi}^{\prime}{ }_{k}
\end{array}\right] \text { and } \bar{\Delta}_{k+1}^{\prime}=\left[\begin{array}{cc}
1 & 0 \\
-\bar{\Delta}_{k} D_{k}^{+} \bar{\pi}^{\prime}{ }_{k} C_{k+1} & \bar{\Delta}^{\prime}{ }_{k}
\end{array}\right] \text {. }
$$

In particular,

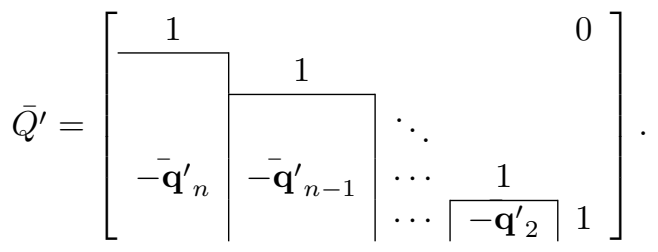

From the form of $\bar{Q}^{\prime}$ we see that $\bar{Q}^{\prime}=I-\left(I-D D^{+}\right) \bar{Q}^{\prime} C D^{+}$, in which $D D^{+} \bar{Q}^{\prime}=D D^{+}$ (Here $D$ is labeled backwards!). This gives $\bar{Q}^{\prime}\left(I+C D^{+}\right)=I+D D^{+} C D^{+}$from which we see that $\bar{Q}^{\prime}=\left(I+D D^{+} C D^{+}\right)\left(I+C D^{+}\right)^{-1}$. Since $M=D+B=D+C$ (with column partitioning and reverse numbering), we can identify these matrices as 
$\bar{Q}^{\prime}=W V^{-1}$, where $W=I+D D^{+} C D^{+}$and $V=I+C D^{+}$.

Lastly, if $U=I+D^{+} C=\left[\begin{array}{cc}? & ? \\ ? & U_{k}\end{array}\right]$ then

$$
U_{k+1}=\left[\begin{array}{cc}
1 & 0 \\
D_{k}^{+} C_{k+1} & U_{k}
\end{array}\right]
$$

and consequently,

$$
U_{k+1}^{-1}=\left[\begin{array}{cc}
1 & 0 \\
-U_{k}^{-1} D_{k}^{+} C_{k+1} & U_{k}^{-1}
\end{array}\right]
$$

On the other hand, we may match this with 6.4 in which $U_{2}^{-1}=\Delta_{2}$. Note that this uses $D_{k}^{+} \bar{\pi}_{k}^{\prime}=D_{k}^{+}$! We have thus shown that $U^{-1}=P^{\prime}$.

\section{Remarks}

(i) The "horizontal" reductions $V^{-1} M U^{-1} Z$ and $W V^{-1} M U^{-1}$, respectively, correspond to the "row" and "column" partitioned cases.

(ii) Since $V^{-1} M U^{-1} Z=W V^{-1} M U^{-1}=D+Y=D+\bar{Y}$, we see that we have two sets of consistency conditions for $M \sim D$, i.e., $Y=0$ and $\bar{Y}=0$. That is,

$$
Y_{k}=\left(1-A_{k} A_{k}^{+}\right) B_{k} \pi_{k-1}\left(I-D_{k-1}^{+} D_{k-1}\right)=0, k=2, \ldots, n
$$

and

$$
\bar{Y}_{k}=\left(1-D_{k-1} D_{k-1}^{+}\right) \bar{\pi}_{k-1} C_{k}\left(I-A_{k}^{+} A_{k}\right)=0, k=2, \ldots, n .
$$

These respectively correspond to the rows or columns of $Y$ being zero.

In conclusion, let us return to Theorem 4.1.

Consider

$$
\bar{N}^{\prime}=\left[\begin{array}{cc}
? & ? \\
? & \bar{N}^{\prime}{ }_{k}
\end{array}\right]
$$

and $\bar{R}_{k}$ as given in 6.2. We may again apply Lemma 3.4 together with $\bar{R}_{k} A_{k}^{+}=0$ and $\bar{N}_{k-1}^{\prime+} \bar{R}_{k}=0$, to obtain a reflexive inverse inverse $\bar{N}_{k}^{\prime}$ such that

$$
\bar{N}_{k}^{\prime}{ }_{k}^{+} \bar{N}_{k}^{\prime}=\left[\begin{array}{cc}
A_{k}^{+} A_{k}+\left(I-A_{k}^{+} A_{k}\right) \bar{R}_{k}^{+} \bar{R}_{k} & 0 \\
0 & \bar{N}_{k-1}^{\prime+} \bar{N}_{k-1}^{\prime}
\end{array}\right]
$$

It now follows by induction, that if $\left(\bar{N}^{\prime}\right)_{k-1}^{+} \bar{N}^{\prime}{ }_{k-1}$ is diagonal, then so is $\bar{N}^{\prime}{ }_{k} \bar{N}^{\prime}{ }_{k}$, and has the form

$$
\begin{gathered}
{\overline{N^{\prime}}}_{k}{ }_{k} \bar{N}^{\prime}{ }_{k}=\operatorname{diag}\left[A_{k}^{+} A_{k}+\left(I-A_{k}^{+} A_{k}\right) \bar{R}_{k}^{+} \bar{R}_{k}, \ldots, A_{2}^{+} A_{2}+\left(1-A_{2}^{+} A_{2}\right) \bar{R}_{2}^{+} \bar{R}_{2}, A_{1}^{+} A_{1}\right] \\
\text { for } k=1, \ldots, n .
\end{gathered}
$$


When $k=n$, we arrive at $\overline{\mathfrak{D}}=\bar{N}_{n}^{\prime+} \bar{N}_{n}^{\prime}$, as desired.

As before, because the product of $A_{k}^{+} A_{k}$ and $I-A_{k}^{+} A_{k}$ is zero, we see that

$$
R S\left[A_{k}^{+} A_{k}+\left(1-A_{k}^{+} A_{k}\right) \bar{R}_{k}^{+} \bar{R}_{k}\right]=R S\left(A_{k}^{+} A_{k}\right) \dot{+} R S\left[\left(1-A_{k}^{+} A_{k}\right) \bar{R}_{k}^{+} \bar{R}_{k}\right]
$$

as an internal direct sum, and hence that

$$
\left.R S\left(A_{k}\right)=R S\left(A_{k}^{+} A_{k}\right) \subseteq R S\left[A_{k}^{+} A_{k}+\left(1-A_{k}^{+} A_{k}\right) \bar{R}_{k}^{+} \bar{R}_{k}\right)\right] .
$$

This allows us to conclude that

COROLlary 6.3.

$$
R S(\bar{D})=\left[\begin{array}{c}
R S\left(A_{1}\right) \\
\vdots \\
R S\left(A_{n}\right)
\end{array}\right] \subseteq\left[\begin{array}{c}
R S\left[A_{n}^{+} A_{n} \dot{+}\left(1-A_{n}^{+} A_{n}\right) \bar{R}_{n}^{+} \bar{R}_{n}\right] \\
\vdots \\
R S\left[A_{2}^{+} A_{2} \dot{+}\left(1-A_{2}^{+} A_{2}\right) \bar{R}_{2}^{+} \bar{R}_{2}\right] \\
R S\left(A_{1}^{+} A_{1}\right)
\end{array}\right]=R S(\overline{\mathfrak{D}})
$$

We may now combine Corollaries 3.2 and 6.3 to conclude that

(i) $R S(\bar{D}) \subseteq R S(\overline{\mathfrak{D}})$

(ii) $R S(\bar{M}) \cong R S\left(\bar{N}^{\prime}\right)=R S\left[\bar{N}^{\prime}+\bar{N}^{\prime}\right]=R S(\overline{\mathfrak{D}})$

(iii) If $\bar{M} \approx \bar{D}$ then $R S(\bar{D}) \cong R S(\bar{M}) \cong R S(\overline{\mathfrak{D}})$.

Now if $R_{q \times q}$ is finite, then by Theorem 1 of [10], we may conclude that $R S(D)=$ $R S(\overline{\mathfrak{D}})$ and thus $R S\left[\left(1-A_{k}^{+} A_{k}\right) \bar{R}_{k}^{+} \bar{R}_{k}=0\right.$. This means that $\bar{R}_{k}=\bar{R}_{k} \bar{R}_{k}^{+} \bar{R}_{k}=0$, for $k=1,2, \ldots, n$ and we have the dual Roth Consistency Conditions $\bar{R}=0$, i.e.,

$$
\bar{R}_{k}=\left(I-\bar{N}_{k-1}^{\prime} \bar{N}_{k-1}^{\prime+}\right) \bar{\pi}_{k-1}^{\prime} C_{k}\left(1-A_{k}^{+} A_{k}\right)=0,
$$

with reverse numbering.

As before, we can use the 'barred' version of Lemma 4.3 to show that these are equivalent to the simpler consistency conditions that $\bar{Y}_{k}=0$ of 6.7 .

7. Questions. We close this section with several open questions.

1. Can we improve on the matrix consistency conditions of 5.2 in terms of matrix $M$ ?

2. Can we use the above to derive consistency conditions for the Stein equation?

3 . What is the block form of $N^{+} N$ ? Can we use induction?

4. Are there any other applications of the "bar" lemma?

5. What horizontal consistency conditions does the second canonical form correspond to?

6. In Theorem 6.2 do two factors suffice?

Acknowledgement. This research was supported by CMAT - Centro de Matemática da Universidade do Minho, Portugal, and by the Portuguese Foundation for Science and Technology - FCT through the research program POCTI. 


\section{REFERENCES}

[1] P. Ara, private communication.

[2] P. Ara, K.R. Goodearl, K.C. O'Meara and E. Pardo, Diagonalization of matrices over regular rings. Linear Algebra and Its Applications, 265:147-163, 1997.

[3] P. Ara, K.R. Goodearl, K.C. O'Meara and E. Pardo, Separative cancellation for projective modules over exchange rings. Israel Journal of Mathematics, 105:105-137, 1998.

[4] J.K. Baksalary and R. Kala, The matrix equation $A X-Y B=C$. Linear Algebra and Its Applications, 25:41-43, 1979.

[5] A. Ben-Israel and T.N.E. Greville, Generalized Inverses: Theory and Applications, Wiley, New York, 1974.

[6] R.M. Guralnick, Roth's theorems and decomposition of modules. Linear Algebra and Its Applications, 39:155-165, 1980.

[7] W.H. Gustafson, Roth's theorems over commutative rings. Linear Algebra and Its Applications, 23:245-251, 1979.

[8] D. Handelman, Perspectivity and cancellation in regular rings. Journal of Algebra, 48:1-16, 1977.

[9] R.E. Hartwig, Block Generalized Inverses, Arch.Rat. Mech Anal, 61(3): 13-44, 1976.

[10] R.E. Hartwig, Roth's equivalence problem in unit regular rings. Proc. Amer. Math. Soc., 59:39-44, 1976

[11] R.E. Hartwig and J. Luh, On finite regular rings. Pacific Journal of Mathematics, 69(1):73-95, 1977.

[12] R.E. Hartwig, P. Patrício and R. Puystjens, Diagonalizing triangular matrices via orthogonal Pierce decompositions, Linear Algebra and its Applications, 401:381-391, 2005.

[13] M. Henriksen, On a class of regular rings that are elementary divisor rings. Archiv der Mathematik, 24:133-141, 1973.

[14] D. Huylebrouck, The generalized inverse of a sum with a radical element: Applications. Linear Algebra and Its Applications, 246:159-175, 1996.

[15] P. Patrício and R. Puystjens, About the von Neumann regularity of triangular block matrices. Linear Algebra and Its Applications, 332-334:485-502, 2001.

[16] W.E. Roth, The equations $\mathrm{AX}-\mathrm{YB}=\mathrm{C}$ and $\mathrm{AX}-\mathrm{XB}=\mathrm{C}$, in matrices, Proc. Amer. Math Soc. 3: 392-396 (1952); MR 13,900 